\title{
Effects of Halothane on Dimyristoylphosphatidylcholine Lipid Bilayer Structure: A Molecular Dynamics Simulation Study
}

\author{
Kwang Jin $\mathrm{Oh}^{*}$ and Michael L. Klein ${ }^{\dagger, a}$ \\ Supercomputing Center, Korea Institute of Science and Technologv Information. Daejeon $305-806$. Korea \\ ${ }^{*}$ E-mail: kohtakisti.re.kr \\ †Center for Molecular Modeling and Department of Chemisty. Unversity of Pennswania. 231S. 3 th st.. \\ Philadelphia, PA 1910+6323, LSA \\ Received June 14, 2009, Accepted Julv 16, 2009
}

\begin{abstract}
We performed molecular dymanics simulations on dimyristoylphosphatidylcholine lipid bilayer with 50 mol\% halothane. The structural properties, electron density profile, segmental order parameter of acyl chains, headgroup orientation distribution, water dipole orientation distribution, have been examined. Overall the effects of the halothane molecules on structural properties of DMPC lipid bilayer were found to be small. The electron density profiles, the segmental order parameter, the headgroup orientation, the water dipole orientation were not affected significantly by the halothane molecules. Pressure tensor calculations shows that the lateral pressure increases at the hydrocarbon tail region and the headgroup region, and decreases at the water-headgroup interfacial region.
\end{abstract}

Key Words: Molecular dỵnamics simulation. Lipid bilaỵer. Anesthesia

\section{Introduction}

Many volatile compounds, when inhaled. induce transient analgesia. amnesia. and immobility in response to a noxious stimulus. This larye and chemically diverse group of compounds is known as inhaled general anesthetics. However molecular mechanism of general anesthesia is still largely unknown. For example. it is still under debate concerning whether anesthetics produces their effects by acting on lipids or proteins. ' Lipid theories postulate that anesthetics acts wia some perturbation of the membrane lipids. whereas protein theories propose that anesthetics acts by interacting with a protein site in the membrane lipid.

To address the problem. many computer simulation studies on models of lipid membranes have been done since computer simulation yield information on the effects of the anesthetics in atomistic details for a given potential model. ${ }^{2-6}$ Due to the difficulty in simulating lipid bilayer system with halothane molecules and protein to trace the effects of the halothane molecules on protein structure. most studies have been devoted to simulate lipid bilayer system with the halothane molecules to address the problem indirectly. So far. several structural properties have been examined from simulations. Those include area per lipid and d-spacing. electron density profile. segment order parameter of acyl chains, headgroup orientation, electrostatic potential difference relative to the bilayer center. distributions of anesthetic molecules in lipid bilayer.

Tu et $\mathrm{al}^{2}$ found a small lateral expansion and a modest contraction in the bilay'er thickness in a molecular dy namics simulation (MD) of dipalmitoylphosphatidylcholine (DPPC) with a mole fraction of $6.5 \%$ halothane. The same behavior was observed in a MD study on DPPC with higher halothane mole fraction (a mole fraction of $50 \%$ halothane) by Koubi et $a l^{3}$

"Present address: Department of Chemistry, Temple University, 1900 North 12th street, Philadelphia, PA 19122, USA.
Koubi et al. ${ }^{2}$ found that overall electron density profile peaks in DPPC with halothane molecules were located around $-17.0 \AA$ and $16.0 \AA$ compared to $-19.0 \AA$ and $8.8 \AA$ for pure DPPC. Although the result was in apparent disagreenent with experimental finding by Franks and Lieb, the changes oberserved in the study by Koubi et al. were likely to fall within the resolution of the experimental data.

Tu et al $^{2}$ found no significant change in the hydrocarbon chain conformations in their MD. However. Koubi et al. ${ }^{3} \mathrm{ob}$ served a decrease in the acyl chain segment order parameters for the tail portion, and a slight increase for the upper portion compared to neat bilayers. which was in agreement with several NMR studies ${ }^{8,9}$ on related sy stems.

Koubi et al. ${ }^{3}$ observed a shift and broadening of the choline headgroup dipole orientation distribution and significant changes in the electrostatic potential difference relative to the bilayer center. The halothane molecules were found to preferentially segregate to the upper part of the lipid acyl chains with a maximum probability near the $C_{5}$ methylene groups. However a finite probability was also observed along the tail region and across the methyl trough.

Recently, there have been interesting simulation studies by Koubi $e t a l^{4,5}$ They performed a MD simulation on dinyristoylphosphatidylcholine (DMPC) lipid bilayer with "nonimmobilizer $C_{2} F_{6}$. The noninmobilizers are molecules which do not suppress movement. but cause amnesia. Due to structures and lipophilicities similar to those of known inhaled anesthetics. the noninmobilizers were predicted to be good anesthetics, but were found to lack anesthetic properties. They found that the overall structure of the lipid bilayer. acyl chain conformations. and the zwitterionic headgroup dipole orientation undergo only a slight modification compared with the pure lipid bilayer with virtually no change in the potential across the interface. In particular, the nonimmobilizer molecules were evenly distributed along the lipid hydrocarbon chains with a slight preference for the bilayer center. Another intere- 
sting study has been done by Tang and $\mathrm{Xu} .{ }^{6}$ They reported 2.2-ns MD simulation on DMPC lipid bilayer with a gramicidin channel and halothane. whose concentration was comparable to that at clinical condition. They found that anesthetics preferentially targeted the anchoring residues at the channel-lipid-water interface. Although the anesthetic effect on channel structure was minimal. it was found that the presence of halothane profoundly affected chanuel dy namics.

The purpose of this study is to investigate the effects of halothane on lipid bilayer structure and gain an insight into molecular mechanism of anesthesia. We performed a MD simulation on DMPC lipid bilayer. The concentration of halothane was set to a mole fraction of $50 \%$ halothane to enliance the halothane effects.

\section{Molecular Dynamics Simulations}

We performed two simulations using a general purpose molecular dynamics simulation program. ${ }^{34}$ One is pure dimyristoy lphosphatidy lcholine (DMPC) lipid bilayer simulation and the other is the lipid bilayer simulation with 2-bromo-2-chloro1.1.1-trifluoroethane (halothane) for general anesthetics. See figure 1 for the chemical structures of DMPC and halothane. Our system contains 64 DMPC and 1645 water in a periodic box and additional 32 halothane molecules in the pure lipid bilayer system for the latter simulation. The lipid bilay'er composition corresponds to a fully hydrated DMPC lipid bilayer at $303 \mathrm{~K}$ with water per lipid molecule. $n_{w}=25.7$. The dimension of the box was set to $48.6 \times 38.7 \times 62.0 \AA^{3}$. The simulations were performed in the lipid liquid cry stalline phase $\left(L_{1}\right)$. This phase is exhibited above $\mathrm{T}=297 \mathrm{~K}$ for DMPC. The most accurate experimental data on the biorelevant hydrated $L_{\omega}$ phase of DMPC have been obtained at $\mathrm{T}=303 \mathrm{~K}$ for DMPC. Note that the lipid/halothane ratio is $2: 1$. This ratio corresponds to a mole fraction of $50 \%$ and is higher than clinically relevant concentrations. But the elevated concentration of anesthetic. compared with clinical application, has enabled us to efficiently sample the halothane distribution and determine its effect on the membrane structure.

We used a popular biomolecular force field $\mathrm{CHARMM} 27^{10}$ for DMPC and the TIP3P model ${ }^{11}$ for water. and finally force field parameters for halothane optimized by Scharf and Laasonen. ${ }^{12}$ A Verlet neighbor list scheme combined with celllinked list was used for short-ranged nonbonded interactions. Here. the potential cutoff distance was set to $10 \AA$ and the Verlet skin-depth was set to $2.0 \AA$. To avoid the discontinuity in force due to the potential truncation, we used a switching finction. Electrostatic interactions were handled by the particle mesh Ewald method ${ }^{13}$ with tin-foil boundary conditions. The Ewald parameters and $\mathrm{k}_{\text {max }}$ were set to 0.3 and 32 . respectively. For production run. simple potential truncation of electrostatic interactions was used because pressure profile calculation combined with particle mesh ewald is not feasible.

The equations of motion were integrated using an algorithm based on the reversible multiple time step technique. ${ }^{1.15}$ The temperature was maintained using the Nose-Hoover chain method. ${ }^{34}$ One Nose-Hoover chain of 5 themostats was connected to the system. The integration time step was set to $2 \mathrm{fs}$
Dimyristroy|phosphatidylcholine (DMPC)

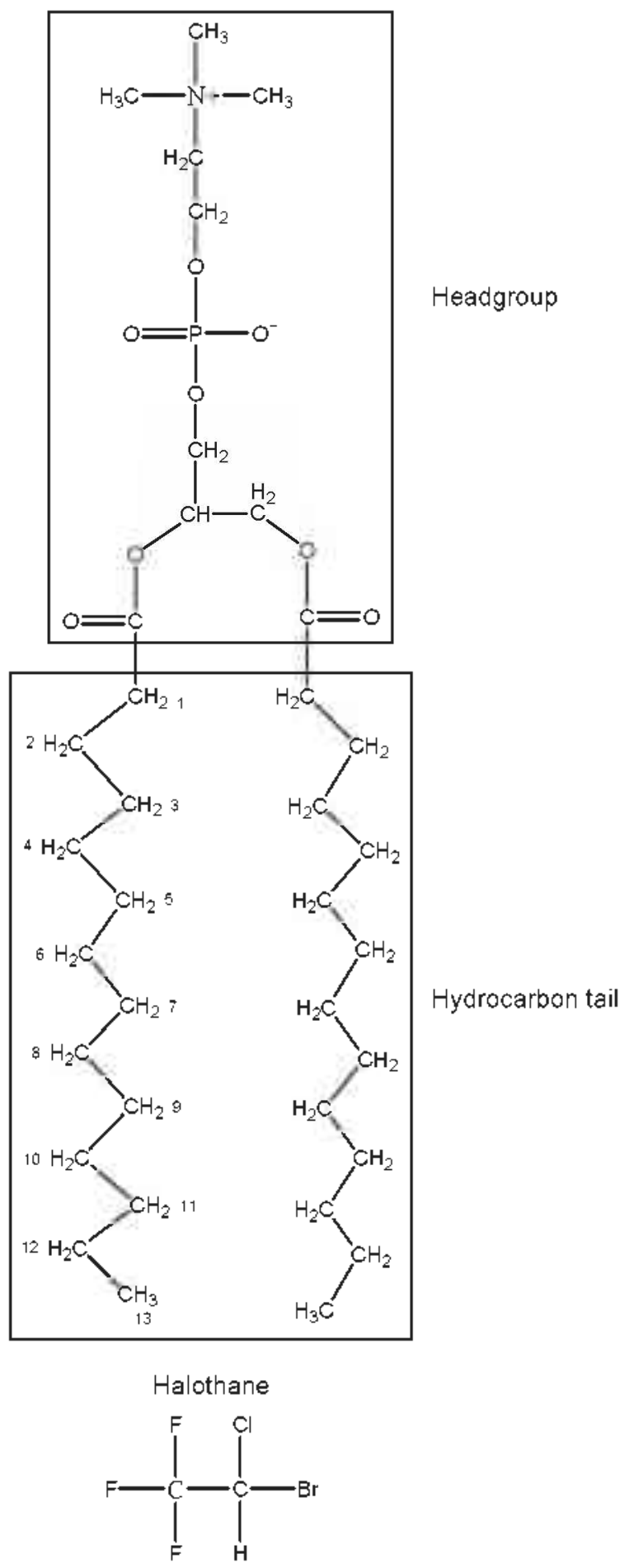

Figure 1. Chemical structure of dimyristoylphosphatidylcholine (DMPC) and halothane molecule. The headgroup and the hydrocarbon tail are shown in rectangles. The carbon number of acyl chains are also shown in the figure.

after an initial preliminary run and the thermostat mass was set to $1000 \mathrm{fs}$. All bonds containing hydrogen atonts were constrained to their equilibrium value by the SHAKE/RATTLE algorithm. ${ }^{16,1 ?}$ A tolerance for the SHAKE/RATTLE iteration was set to $10^{-6}$. 

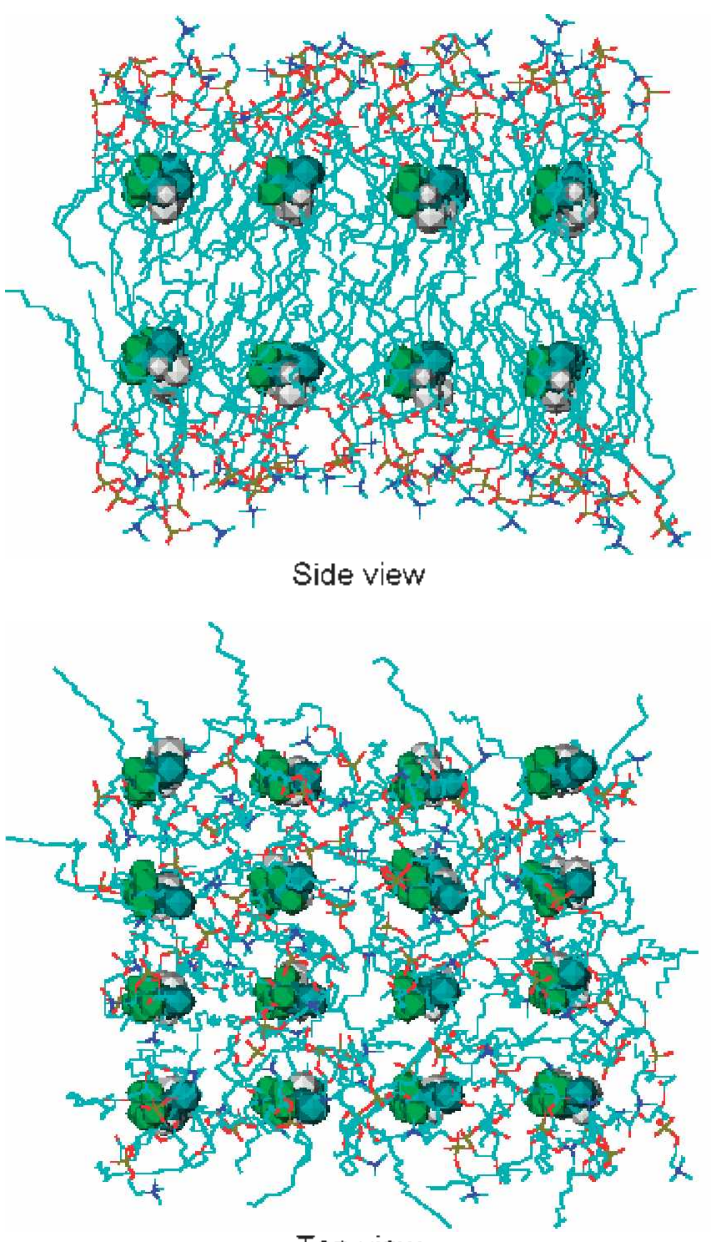

Top view

Figure 2. Initial setup for lipid bilayer simulation with halothane molecules. 16 halothane molecules, which are distributed on $x-y$ plane, were placed in the equilibrated lipid bilayer sample at about $8 \AA$ above and below from the bilayer center $z=0$. Water and hydrogen atoms in the lipid molecules are not shown in the tigure. Atoms in the halothane molecules are represented by van der Waals sphere.

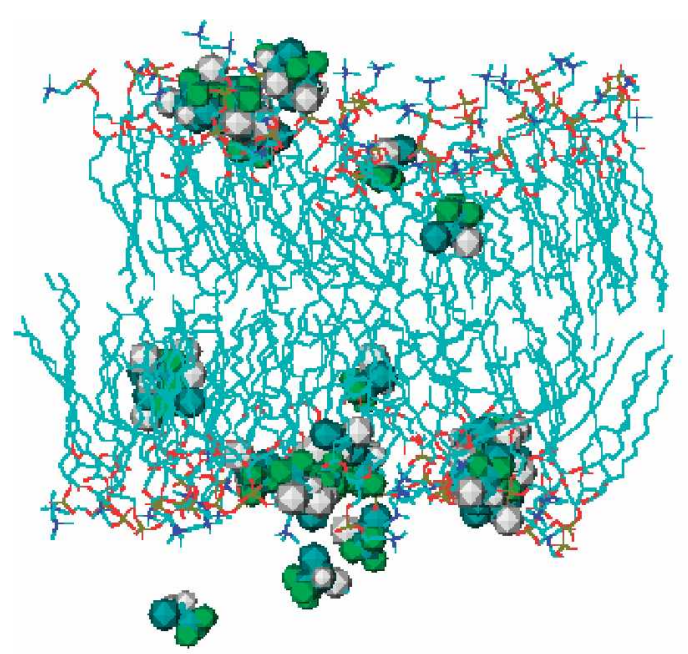

Figure 3. Snapshot trom lipid bilayer simulation with 32 halothane molecules. Water and hydrogen atoms in the lipid molecules are not shown in the figure. Atoms in the halothane molecules are represented by van der Waals sphere
The initial configuration for lipid bilayer sinulation without halothane molecules was obtained from the previous simulation by Koubi $e t a l^{4}$ The initial configuration for lipid bilayer simulation with halothane molecules was prepared as follows. First. 16 halothane molecules. which are distributed on $x-y$ plane. were placed in the equilibrated lipid bilayer sample at $8 \AA$ above and below from the bilayer center $z=0$ (see Figure 2). Then the whole system was numimized using steepest descent method to remove bad contacts. After nininization we equilibrated the system for about 1.8 nanoseconds. Data for analy sis were collected from further about 5 nanoseconds simulation after equilibration. which is longer simulation than previous simulations by $\mathrm{Tu}$ et $a l^{2}$ and Koubi et al. ${ }^{3.4 .5}$ Figure 3 shows typical snapshot from the equilibrated configurations.
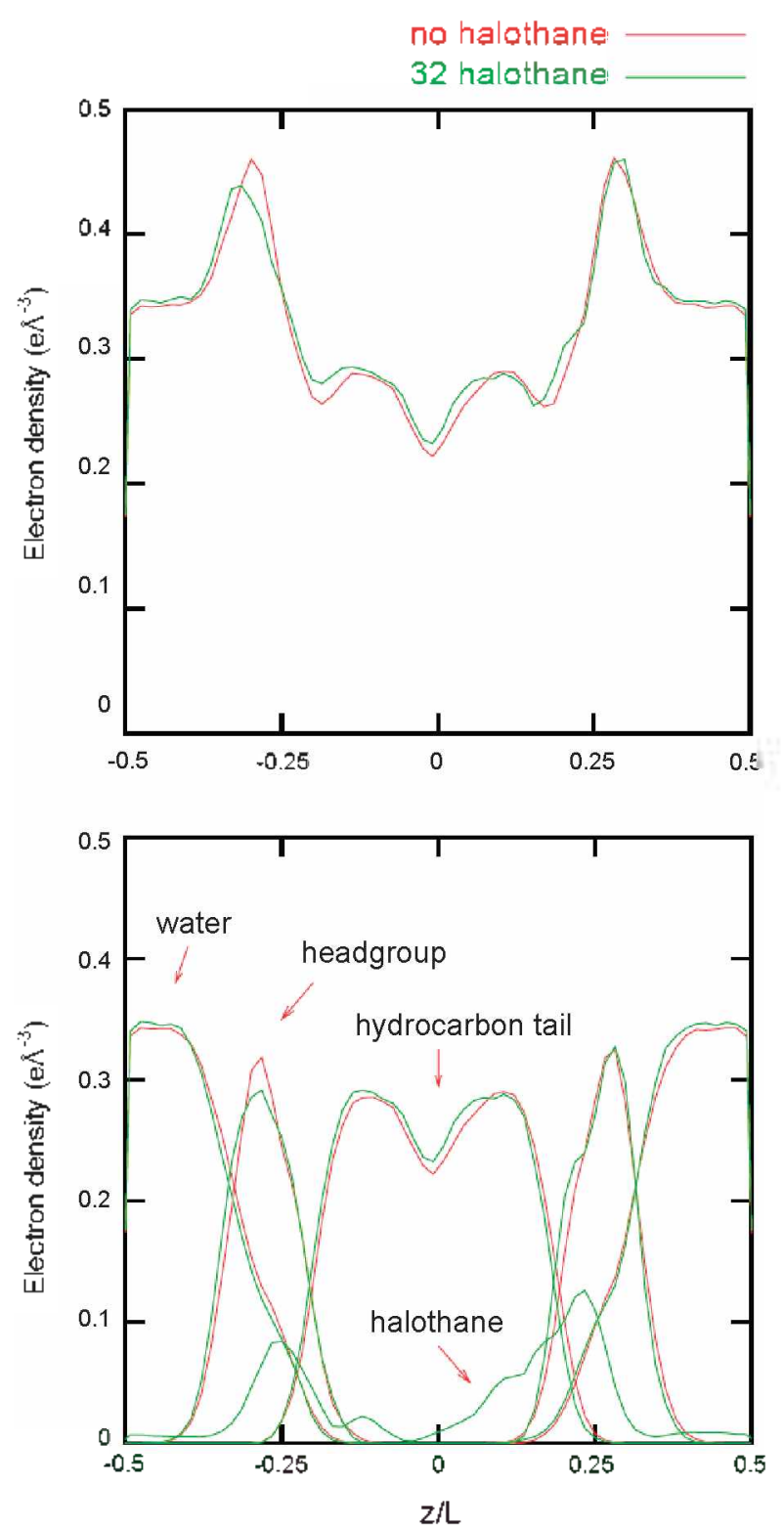

Figure 4. Total electron density profile (upper plot) and its components (lower plot) from the headgroups, the hydrocarbon tails, and water. The contribution from the halothane molecules is also shown in the lower plot, but not included in the total electron density profiles. 


\section{Results and Discussion}

Figure + shows total electron density profiles from the two simulations. The electron density profiles contributed from three components i.e.. the headgroups. the hydrocarbon tails. and water. were also shown. For the simulation with halothane. the electron density profile from the halothane molecules was also shown in the Figure. but not included in the total electron density profile calculation to compare electron density profiles only from the three components. Here the electron density profiles were calculated by dividing simulation box into thin slabs along the bilayer normal and counting the number of electrons on each slab. The slab thickness was set to $1 \mathrm{~A}$.

The electron density profiles show two small minima at the interfacial regions between the headgroups and the hydrocarbon tails. Those were not observed in previous simulations on DMPC. But the electron density profiles are consistent with those observed in other lipid bilayer systems. That is to say. the electron density profiles show the two peaks from the headgroups and a large minimum at the bilayer center.

From the Figure, it is evident that the effects of halothane molecules on the electron density profile are small. For example. the locations of the peaks in the density profile from pure lipid bilayer. which were located at the headgroup regions. were not changed significantly by the halothane molecules. This result is in agreement with X-ray results by Franks and Lieb. even in the presence of anesthetic at high concentration up to a mole fraction of $60 \%$ halothane. The different result from us and the experimental result was obtained in DPPC lipid bilayer simulation study with halothane of $50 \mathrm{~mol} \%$ by Koubi et $a l^{4}$ such that the peaks in total electron density profile were observed at different locations from those in pure DPPC lipid bilayer. However, this discrepancy does not mean any inconsistency with the X-ray result because the changes observed in the DPPC lipid bilayer system are likely to fall within the resolution of the $\mathrm{X}$-ray data.

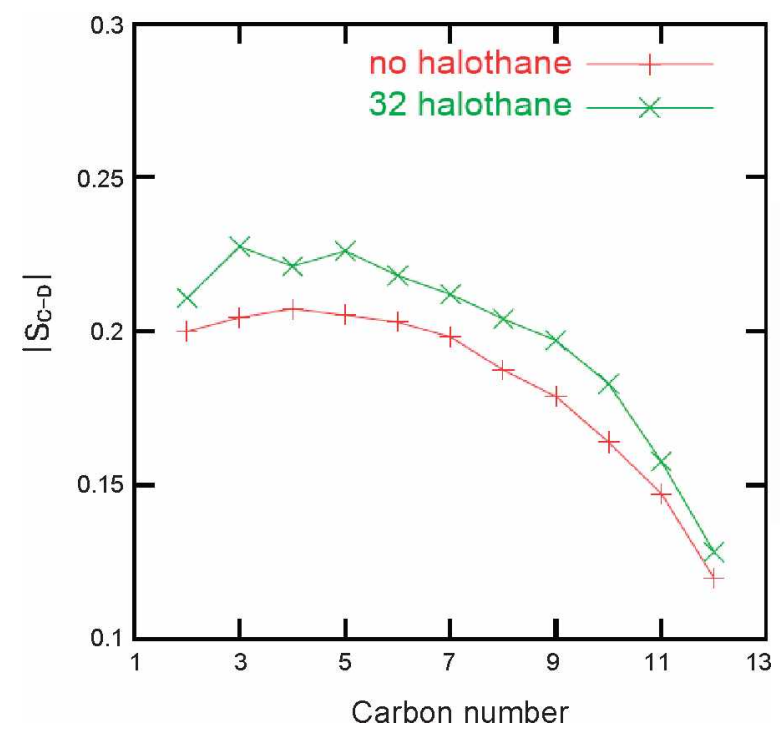

Figure 5. Deuterium order parameter $\mid \mathrm{S}$ co 0 of the acyl chain as a finction of carton nuniber in the hydrocarton tail. The carbon numbers are shown in Figure 1.
The location of the halothane molecules in the lipid bilayer is also of interest. A number of experimental results on relatively simple model lipid șistems have showed discrepancy as to the locations of the halothane molecules in the lipid. According to those results. the halothane molecules have been proposed to distribute in the acyl chain donain, ${ }^{18}$ the headgroup region. ${ }^{8.9}$ or water/lipid interfacial region. ${ }^{19-\hat{-}]}$ In our case the peaks by the halothane molecules in the electron density profile are located in the headgroup regions as shown in the figure. Thus the distribution of halothane molecules is mainly concentrated on the headgroup regions. This is in good agreement with the previous simulations "and experimental results. ${ }^{89}$

Figure 5 shows deuterium order paraneter $\left|\mathrm{S}_{\mathrm{D}}\right|$ as a function of carbon number. which is averaged over $\mathrm{MD}$ trajectory. Here the $S_{C D}$ is given by $S_{C D}=<3 \cos ^{2} \theta-1>/ 2$ where $\theta$ is the angle between the normal to the bilayer and the normal to the plane of the two C-D bonds in a deuterated methylene group of the lipid acyl chain. An $S_{C D}$ value of 1 indicates that the chains are all trans and perpendicular to the bilayer plane. a value of -0.5 indicates that they are all trans and parallel to the plane, and 0 indicates random orientation. We carried out sinulation with explicit hydrogens along the lipid chains and not for deuterated lipids. Thus we assumed that the configurations of the deuterated lipids are identical to those with explicit hydrogens.

The segmental orders of acyl chains show higher values for the upper segments (segments close to the headgroup) as observed in other lipid bilayer simulations. When the segmental orders of the acyl chains from pure lipid bilayer simulation were compared to those from lipid bilayer simulation with halothane, the segmental orders of the acyl chains show slightly highervalues at all segments than the ones from the pure lipid bilayer sinulation as shown in the figure. This behavior is different from the behavior observed in previous DPPC lipid bilayer simulation " with a mole fraction of $50 \%$ halothane. In the DPPC lipid bilayer simulation the ratio of order parameter was greater than 1 for the upper segments of the acyl chains

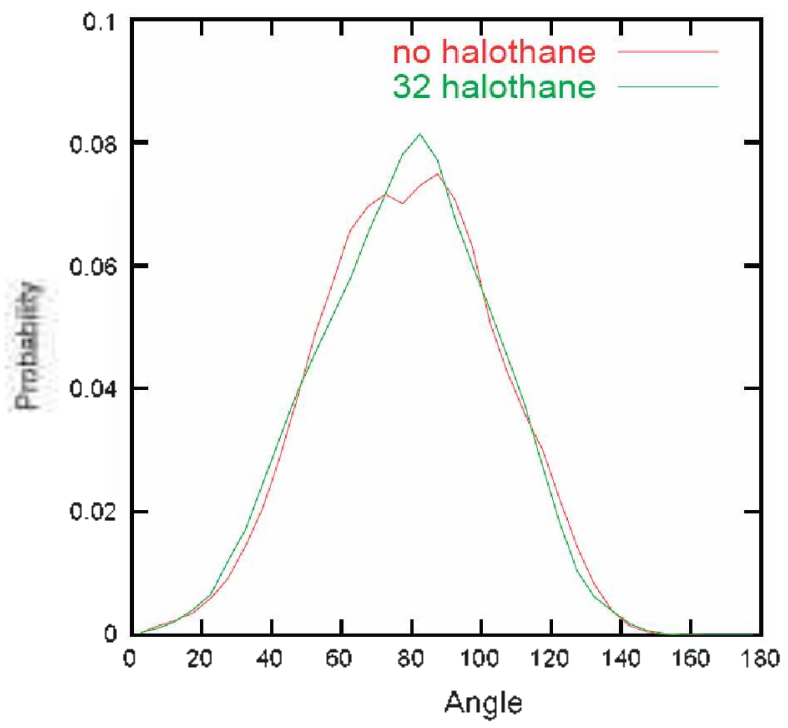

Figure 6. Probability distribution of the angle between the unit distance vector pointing from $\mathrm{P}$ atom to $\mathrm{N}$ atom with respect to the bilaver nomal. 
and decreases significantly at the end of the acyl chain. Neverthless. the overall change due to the halothane molecules is within about $10 \%$. Note that NMR study on palmitoy loleoylphosphatidy lcholine bilayer reported no significant change in the segment order of the acyl chains at low anesthetic concentration. The reported change of the order parameters was less than $10 \%$ up to a concentration of $40 \mathrm{~mol} \%$ of halothane.

Figure 6 shows the probability distribution of the angle between the unit distance vector pointing from $\mathrm{P}$ atom to $\mathrm{N}$ atom with respect to the bilayer normal. which describes the headgroup orientation distribution. The probability distributions were calculated by dividing simulation box into thin slabs along the bilayer nomal and counting the frequency of the angle on each slab. The slab thickness was set to $5^{\circ}$.

The probability distribution in the pure DMPC lipid bilayer shows small double peaks around $75^{\circ}$, but not well separated. In the presence of the halothane molecules. the peaks were merged into single peak, which is located at about $80^{\circ}$, and ligher. However the changes in the probability distribution due to the halothane molecules are not significant in a sense that the calculated average angles were not quite different each other. The obtained values were $79.1^{\circ}$ and $78.5^{\circ}$ for the former and the latter, respectively. Thus we can see that the headgroup orientation is not affected significantly' by the halothane molecules. From the average values. we can also see that the headgroups are oriented almost parallel to the bilayer plane (about $10^{\circ}$ above the bilayer plane). The parallel alignment of the headgroups with the bilayer plane is in good agreement with experimental findings ${ }^{22}$ as well as previous simulations. 2.24

Figure 7 shows the cosine of the angle between the water dipole moment and the bilay'er normal along the bilayer nomal. There is no data at the lipid bilayer core region because water molecules cannot penetrate deeply into the hydrophobic core regions. Water dipole orientations are random at bulk. Therefore the cosine is zero at the bulk region as we can expect. However water molecules gain orientational order due to the electro-

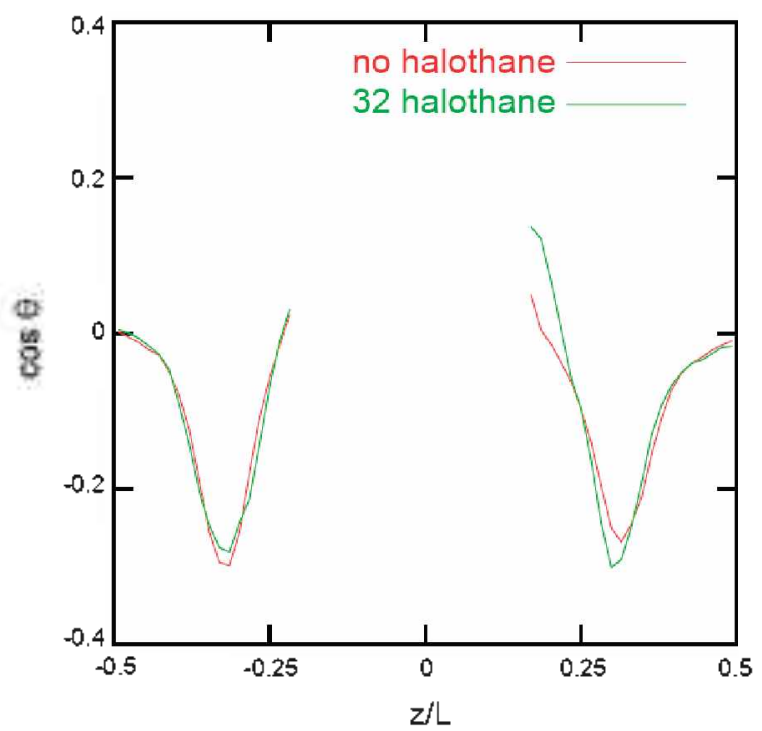

Figure 7. Cosine of the angle between the water dipole moment and the bilaver normal. static interactions as the water molecules approach the headgroup region. As indicated by the negative values of the cosine. the angle between the dipole moments of water molecules and the bilayer normal at the aqueous interfaces is greater than $90^{\circ}$, that is to say, the dipole moments of the water molecules point toward the headgroup region. However we cannot see any apparent changes in water dipole orientation distribution due to the halothane molecules as shown in the figure. The effects of the halothane molecules on the water dipole orientation are also negligible.

Figure 8 shows pressure profile of $\mathrm{P}_{\mathrm{T}}-\mathrm{P}_{\mathrm{N}}$ where $\mathrm{P}_{\mathrm{T}}$ and $\mathrm{P}_{\mathrm{N}}$ are the transverse and normal component of pressure tensor with respect to the bilayer plane. Here the pressure profiles were calculated based on the method by Goetz and Lipowsky. The pressure profile for the compont from $m$-body potential is given by

$$
P^{\alpha \beta(n i)}(z)=-\frac{1}{m\lrcorner I^{r}} \sum\left(\vec{f}_{l}^{\alpha}-\vec{f}_{k}^{\alpha}\right) \vec{y}_{l k}^{\beta} f\left(z_{l}, z_{k}, z\right)
$$

where $\Delta \mathrm{V}$ is the slab volume. $f$ is force acting on particle $l . \vec{r}_{k k}$ is the distance vector between particle / and particle $k . f\left(z_{7}\right.$, $\left.z_{k}, z\right)$ is a weighting factor. The weighting factor $f\left(z_{i}, z_{k}, z\right)$ is chosen such that the fractional contribution of the interaction between a pair of particles / and $k$ to the pressure tensor in the slab centered at $\mathrm{z}$ is $\mathrm{I}$ if both particles are in the slab. $\mathrm{dz} /\left|\mathrm{z}_{\mathrm{f}}-\mathrm{zk}_{\mathrm{k}}\right|$ if one particle is in the slab. $\Delta z / z_{\mid}-z_{k} \mid$ if neither particle is in the slab. but the slab lies between the two particles. Here $\mathrm{d} z$ is the distance of the particle in the slab to the boundary of the slab closet to the other particle and $\Delta z$ is the slab thickness. The number of bins was set to 100 . After pressure profiles were obtained. the pressure profiles were synumetrized and snoothed using 7-point nunning average. Thus this nunuing averaging amounts to choosing a sampling bin thickness of $4.3 \AA$.

Overall features of the pressure profiles are consistent with pressure profiles obtained for other lipid bilayer systems. ${ }^{2}$ i. 27

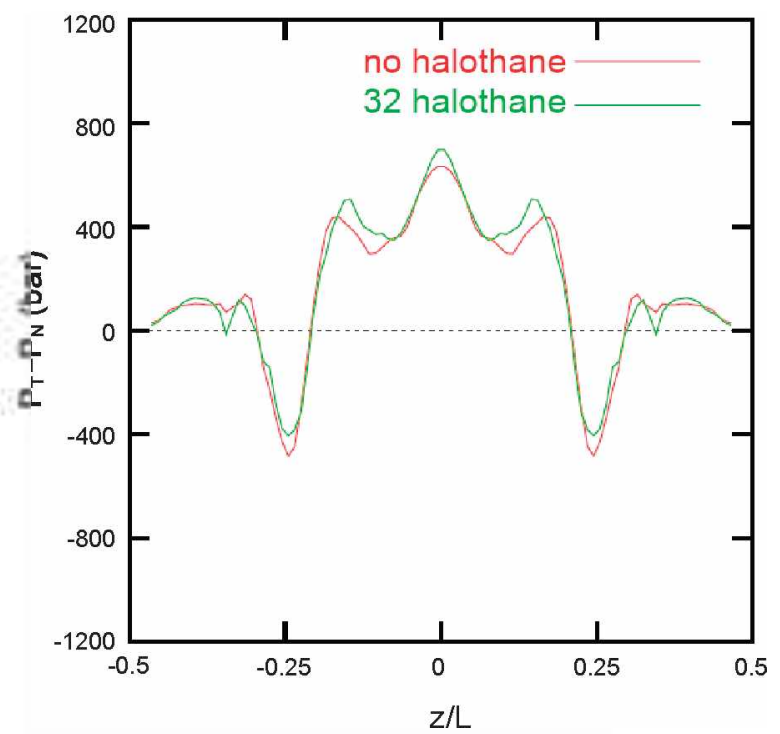

Figure 8. Pressure profile $\mathrm{P}_{\mathrm{T}} \mathrm{P}_{\mathrm{N}}$ where $\mathrm{P}_{\mathrm{T}}$ is transverse component of pressure tensor and $\mathrm{P}_{\mathrm{N}}$ is the normal component of pressure tensor with respect to the bilayer plane. 
The pressure profiles including ours commonly show three positive peaks in the interior region. followed by deep negative peaks. and small positive peaks at the outermost regions. When compared to the electron density profiles, the three positive peaks. two negative peaks. and the outennost positive peaks are located at the hydrocarbon tail region, the headgroup region, and the aqueous region, respectively. Thus because the negative and positive values in the pressure profiles indicate positive and negative surface tensions, respectively. we can see that the headgroup region tends to shrink while the hyddrocarbon tail region and the aqueous region tend to expand.

Figure 8 shows that the increase of three peaks in the hydrocarbon tail region and two deep trough at the headgroup region. and the decrease at $z / L= \pm 0.37$. which approximately corresponds to water-headgroup interfacial region (see Figure 4). Thus the hydrocarbon tail region tends to expand more while the water-interfacial region tends to expand less than before. On the other hand, the headgroup region would feel less surface tension. Note that for interfacially active solutes except long n-alkanols. a large lateral pressure increase is predicted to occur near the aqueous interface. with a compensating decrease near the middle of the bilay'er. ${ }^{3 ! !}$

\section{Conclusion}

We perfonmed two molecular dýnamics simulations. One is pure DMPC lipid bilayer simulation and the other is DMPC lipid bilayer simulation with $50 \mathrm{~mol} \%$ halothane. The structural properties. i.e.. electron density profile. segmental order parameter of acyl chains, headgroup orientation distribution, water dipole orientation distribution, pressure profile were examined to investigate the effects of the halothane molecules on lipid bilayer structure.

From the comparison of the structural properties. we have found that the effects of the halothane molecules on structural properties of DMPC lipid bilayer are overall small. The electron density profiles. the segmental order parameter, the headgroup orientation. the water dipole orientation were not affected significantly by the halothane molecules. Note that the results were obtained at much higher halothane concentration than relevant clinical halothane concentration. Therefore the effects of the halothane molecules would be even smaller at clinical conditions. The present results are in close agreement with the experimental findings by Franks and Lieb ${ }^{-8}$ that the changes in thickness. order parameter profiles, phase transition tempterature are very small at clinically relevant anesthetics concentrations and can be produced in the absence of anesthetic through slight changes in other variables such as temperature.

Cantor ${ }^{29.53}$ postulated that incorporation of anesthetics into the lipid bilayer can produce the redistribution of lateral pressure in the lipid bilayer. which in turn can shift the confonmational equilibrium of transmembrane proteins such as ligand-gated ion channels. For example. if the channel opening involves the increase of the cross-sectional area of the protein at higher positive surface tension region, then the channel opening will be restricted to favor the closed state since the channel opening will require greater work against this higher pressure. Thus Cantor proposed that pressure profile may be such a property that it is strongly affected by incorporation of interfacially active solute as well as by altered lipid composition. but not by small changes in temperature.

Our results partly support Cantor's proposal in a sense that oudr pressure profile were varied in the presence of halothane molecules and the changes in some regions was almost 100 bar. However. further studies should be done to clarify how the variations in pressure profiles affect transmembrane protein conformation as predicted by Cantor. For example. Gullingsnud and Schulten ${ }^{2 / i}$ showed that pressure profiles do not contribute to the discrepancy between measured and modeled changes in the cross-sectional area of MscL channel during gating. but may provide a mechanism for the dependence of MscL gating threshold on lipid composition.

\section{References}

1. Eckenhoff, R. G.: Tohansson, J. S. Phamacol. Rev. 1997, 49, 343.

2. Tu, K.; Tarek, M.; Klein, M. L.; Scharf, D. Biophys. J. 1998, 75, 2123

3. Koubi, L.; Tarek, M; Klein, M. L.; Scharf, D. Biophys. J. 2000, 78,800

4. Koubi, L.; Tarek, M.; Bandyopadhyay, S.; Klein, M. L.; Scharf, D. Biopins. J. 2001, 81, 3339.

5. Koubi, L; Tarek, M.; Bandyopadhyay, S.; Klein, M. L.; Scharf, D. Anesthesiolog, 2002,97, 848 .

6. Tang, P: Xu, Y. Proc. Natl. Acat. Sci. LS. 2002, 99, 16035.

7. Franks, N. P.: Lieb, W. R. J. Hol Biol. 1979, 133, 469

8. Barber, J.: Ellena, T. F.: Cafiso, D. S. Biochemistiv 1995, $34,6533$.

9. North, C.; Catiso, D. S. Biophys. J. 1997, 72, 1754.

10. Feller, S.: Mackerell, Ir., A. D. J. Phus. Chem. B 2000, 104, 7510.

11. Torgensen, W. L.; Chandrasekhar, J.; Madura, T. D.; Impey, R. W.: Klein, M. L. J. Chem. Phus. 1983, 79, 926.

12. Scharf, D.; Laasonen, K. Chem. Phws. Lett 1996, 258, 276.

13. Essmann, U.; Perera, L.: Berkowitz, M. L.; Darden, T.: Lee, H.; Pedersen, L. G. J. Chem. Phys. 1995, 103, 8577.

14. Martyna, G. I.; Tuckerman, M. E.: Tobias, D. I.; Klein, M. L. Hol. Phys. 1996, 87, 1117.

15. Marchi, M; Procacci, P. T. Chem. Phss 1998, 109, 5194

16. Ryckaert, I.-P.; Ciccotti, G.; Berendsen, H. I. C. J. Comp. Chem. $1977,23,327$

17. Andersen, H. C. J. Comp. Phus. 1983, 52, 24.

18. Eckenhott, R. G. Proc. Natt. Acad. Sci. LSA 1996, 93, 2807.

19. Trudell, T. R.: Hubble, W. L. Anesthesiolog, 1976, 44, 202

20. Xu, Y.; Tang, P. Biochim. Biophys. Acta-Biomembranes 1997 , 1323,154

21. Tang, P.; Yan, B.; Xu, Y. Biophis. J. 1997, 72, 1676.

22. Seelig, T.; Macdonald, P. M.; Scherer, P. G. Biochemistrn 1987. 26,7535

23. Bandyopadhyay, S.: Shelley, J. C.: Klein, M. L. J.Phs. Chem. B 2001, 105, 5979.

24. Tu, K.; Tobias, D. T.; Klein, M. L. Biophys. J. 1995, 69, 2558.

25. Goetz, R.: Lipowsky, R. J. Chem. Phs 1998, 108, 7397.

26. Gullingsrud, J; Schulten, K. Biophys. J. 2004, 86, 3496.

27. Lindalhl E.: Edholm, O. J. Chem. Phys. 2000, 113, 3882.

28. Franks, N. P.; Lieb, W. R. Kattwe 1994, $367,607$.

29. Cantor, R. J. Phus Chem. B 1997, 101, 1723.

30. Cantor, R. Biochentistry $1997,36,2339$.

31. Cantor, R. Biophys. J. 1999,79, 2625.

32. Cantor, R. Biophys. J. 2001, 80, 2284.

33. Cantor, R. Biophis. J. 2002,82, 2520 .

34. Oh, K. J; Klen, M. L. Contp. Phys. Conm. 2006, 174, 560

35. Patrá, M.; Karttunen, M.; Hyvonen, M. T.; Falck, E.; Lindquist, P.: Vattulainen, I. Biophis. J. 2003, 84, 3636 . 